\title{
Science historique et protection du patrimoine: de nouveaux concepts à la base de la carte d'inventaire IVS
}

\author{
Développements en Suisse romande
}

\section{Introduction}

Depuis 1984, un inventaire du patrimoine routier de la Confédération est en cours de réalisation. Répertorier des sites isolés (chemins creux ou taillés dans le rocher, bornes, etc...) s'est vite révélé insuffisant et inadapté aux besoins créés par la mutation paysagère actuelle. Découvrir l'origine et les formes historiques des réseaux de chemins qui structurent nos paysages permettait par contre de saisir les lignes directrices du passé. C'est celle-ci qu'il faut s'efforcer d'intégrer aux projets d'aménagements contemporains.

Mais l'émergence de ce patrimoine passait par celle d'une nouvelle science du territoire. Il ne suffit en effet pas de faire travailler ensemble géographes et historiens pour voir aussitôt émerger un espace pluridisciplinaire. Un cheminement, parfois difficile, fut nécessaire. Au bout du compte, la conception du patrimoine à protéger et celle d'une recherche scientifique apte à le révéler apparaissent indissolublement liées.

\section{Des recherches routières au cœur d'une nouvelle approche}

\subsection{Un inventaire fédéral du patrimoine routier}

Selon le mandat reçu de l'Office fédéral de l'Environnement, des Forêts et du Paysage (OFEFP, en allemand BUWAL) dont il dépend, l'IVS est un inventaire des voies de communications anciennes destiné à fournir des bases historiques à l'aménagement du territoire. L'engagement scientifique des collaborateurs de l'IVS se double donc d'interventions pour protéger concrètement les tracés et sites menacés.

\subsection{Des pratiques traditionnelles depassées ou inadaptées}

Archéologues et historiens se sont penchés depuis fort longtemps sur les voies de communications anciennes.
- Les premiers ont focalisé leur attention sur l'époque romaine. Mais les résultats n'ont jamais été assez denses pour permettre, pour ces temps déjà lointains, autre chose qu'un assemblage de segments fort éloignés les uns des autres. La collection des faits n'a ainsi jamais permis d'atteindre le réseau en tant qu'objet d'étude. Les archéologues sont restés en deçà. Dépourvus de contexte, les plus petits indices ont permis la création de nombreuses voies romaines. Tant du point de l'identification des tracés que de celui de leur datation, il faut en revoir aujourd'hui l'interprétation. Beaucoup de vestiges routiers attribués à l'époque romaine (VION 1987/2, 1989/1) sont aujourd'hui souvent datés du 18 e siècle.

- De leur côté, les historiens se sont, dans l'ensemble, plus intéressés aux communications qu'aux chemins. Les archives livrent un matériel abondant sur la circulation et les mouvements commerciaux. Dès lors, les voies anciennes n'ont le plus souvent été qu'un sujet d'histoire économique, au demeurant forcément marginal. "C'est la circulation qui fait la route», affirmait hier Lucien Lefebvre... Dans cette perspective, les routes n'ont que l'importance des flux commerciaux qu'elles drainent ou que celle de la vie urbaine qu'elles alimentent. Les catégories de la recherche historique traditionnelle ont ainsi naturellement prédominé: les faits routiers relevaient au bout du compte des circulations impliquées par des faits économiques, politico-diplomatiques, juridiques, religieux, etc...

Archéologues et historiens n'ont ainsi pas atteint le réseau. Sa forme, sa structure n'ont jamais été réellement au centre des préoccupations. Au mieux, une description posait les conditions d'une géographie historique régionale. Et les géographes, de leur côté, se sont plus intéressés aux lieux habités qu'aux liaisons qui se nouent entre eux. Les premiers sont en effet un peu plus la Borde 19, 1018 Lausanne. 
stables que les secondes et donc plus faciles à appréhender.

\subsection{Le réseau, nouveau sujet des recherches}

Avec l'IVS, le réseau se devait de devenir l'objet central des recherches. Du point de vue historique, un chemin de desserte rurale du $20 \mathrm{e}$ ou du $19 \mathrm{e}$ siècle peut fort bien se révéler être un grand chemin au $17 \mathrm{e}$ ou au 16e siècle. Il est donc impossible de faire un tri à priori dans les recherches à effectuer. Les buts de l'inventaire impliquent ainsi une couverture territoriale complète.

Dès lors, les approches traditionnelles, en laissant de côté le réseau, étaient désarmées pour appréhender des formes complexes. Certains chercheurs récents ont eu conscience de cette inadaptation de l'histoire à aborder les faits géographiques. «Si l'analyse du temps de l'histoire est depuis longtemps particulièrement raffinée, celle de son espace reste en grande partie à venir» (LEPETIT, 1984, p. 120).

Qu'est-ce qu'un réseau routier? Comment l'appréhender? Fernand Braudel disait: «Villes et routes, routes et villes ne sont qu'un seul et même équipement humain de l'espace» (BRAUDEL. 1985, p. 254). Des localités et des liaisons, certes, mais comment penser le mouvement qui les anime? Car un réseau, s'il est souvent soutenu de flux millénaires, est affecté de rapides pulsations conjoncturelles. Aussi convient-il de bien s'entendre sur les termes de flux, itinéraires et tracés trop souvent confondus (VION. 1990/1). Régionalement, la concurrence des villes et le développement des villages ont constamment remodelé les réseaux routiers dont les tracés se sont accumulés dans le paysage (vion, 1989/1). Et le temps de l'espace ne se laisse pas découper comme celui de l'histoire car, écartelées, les logiques spatiales deviennent incompréhensibles (VION, 1989/2). Il faut donc saisir le long terme comme les fluctuations d'une trame polyforme. Il est encore facile de suivre un itinéraire aux nombreux tracés. Mais dix? Mais vingt? Les mêmes tracés peuvent en effet servir à des itinéraires différents, successifs ou non.

Les faits qui attestent le réseau demandent aussi à être interprétés. Et, au regard des errement précédents, il ne saurait être question de se passer de méthodes de lecture. Tous les documents ne peuvent être mis sur le même plan, mais chacun doit trouver sa place dans un ensemble cohérent. Les représentations spatiales (cartes, plans) doivent être confrontées aux mentions d'itinéraires tirés des textes. Il faut savoir estimer la pertinence de ces attestations: l'itinéraire n'est-il plus qu'un souvenir ou la circulation qui l'emprunte est-elle réelle? La forme du réseau est une autre réalité concrète, dont il faut dégager une science morphologique.

\section{La carte d'inventaire IVS}

\subsection{Présentation}

La carte d'inventaire IVS ne présente pas une synthèse historique mais un état du patrimoine à protéger. Ses éléments sont classés dans trois catégories, dites d'importance nationale, régionale et locale, déterminées par la loi fédérale sur la protection de la nature et du paysage.

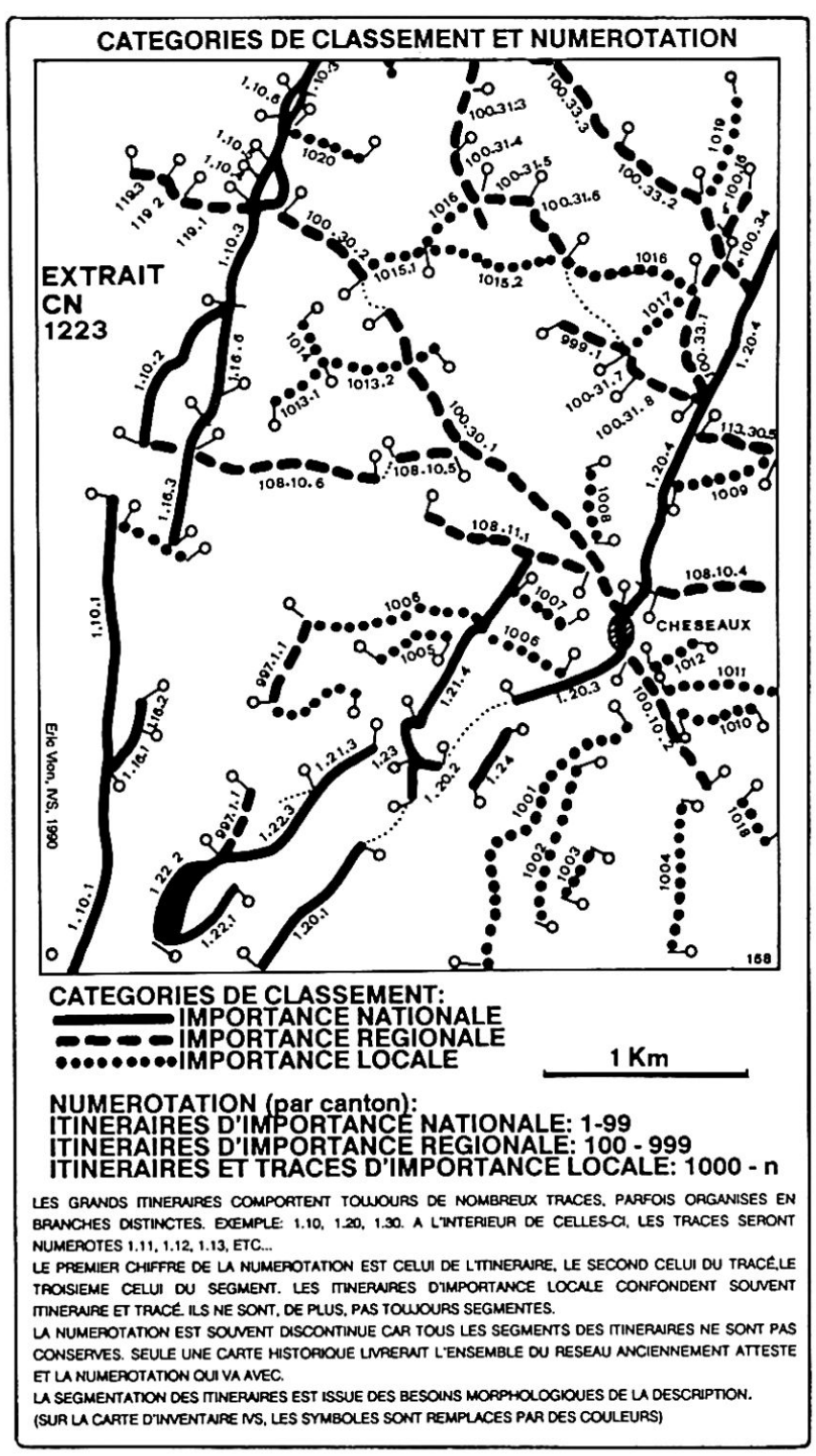

Figure 1 Les tracés protégés tombent dans les trois catégories prévues par la loi fédérale sur la protection de la nature et du paysage: importance nationale, régionale et locale. La numérotation des chemins conservent cette division. On remarquera que l'attestation des itinéraires est discontinu car seuls ceux qui ont subsisté jusqu'à nos jours méritent une protection! La carte d'inventaire IVS est présentée au 1:25000 et en couleur. L'itinéraire d'importance nationale est celui présenté dans la figure 3 (Extrait de la CN 1223 - Echallens). 
La fonction actuelle des éléments qui composent ce patrimoine est très diverse. A côté de vestiges, de sentiers et de chemins ruraux, de grandes routes goudronnées sont inscrites à l'inventaire.

Parce qu'ils sont la composante fondamentale des réseaux, les tracés des itinéraires ont retenu l'attention au premier chef. C'est eux qui assurent la trame de nos paysages en les découpant. Le monument, le site ou le revêtement ne prennent, fondamentalement, leur importance que par rapport aux réseaux formés par les premiers.

La carte IVS n'est pas passéiste et sa rupture avec la carte archéologique traditionnelle est claire. On ne collectionne pas les éléments disparus d'un passé révolu, on livre les racines historiques d'un présent vécu.

Ce n'est donc pas le passé qui importe en premier lieu, c'est la réalité du patrimoine routier actuel. De ce point de vue, le réseau historique est forcément en lambeaux. Pour devenir lisible en tant que tel, il devrait être accompagné d'une carte des itinéraires historiques ou, au moins, de plans particuliers de ceux-ci.

Mais le tout, qui est plus que la somme des parties, peut admettre ces lacunes car elles sont elles-mêmes chargées de sens. Elles signalent en effet les nouveaux aménagements qui les ont impliqué.

L'émergence du réseau dans la protection du patrimoine comme la lecture régressive que l'on en propose sont ainsi tout à fait symétriques et dépendantes de celle qui a vu le jour dans la recherche historique.

\subsection{Trois types de cartes en compétition}

Les recherches d'archives et de terrain devaient s'exprimer dans des résultats. Trois directions cartographiques principales pouvaient être prises.

- La première reste la plus proche des relevés. La prospection de terrain livre des sites. Les relevés d'archives donnent d'abord un réseau, puis au fur et à mesure que l'on remonte le temps, des segments. Ces sites sont transcrits sur la carte au $1: 25000$ et des couleurs peuvent symboliser des époques. Mais comme les faits attestés sont aussi hétérogènes que les sources qui les contiennent, la carte devient vite illisible: la discontinuité des couleurs domine. Son seul intérêt est de fournir des indications claires sur la qualité scientifique des attestations historiques.

- La seconde carte possible est interprétative. L'élaboration des relevés a été effectuée et les différents éléments sont organisés en itinéraires. La hiérarchie et la chronologie des tracés ne sont exprimés, s'il y a lieu, que par des symboles secondaires. C'est ici que le travail de l'histoire des communications trouve sa dimension. De cette carte des itinéraires historiques est issue le suivante:

- La troisième carte est un outil de protection du patrimoine. Elle seule répond aux buts fixés par le mandat de l'IVS. Y figurent les tracés et les sites à protéger. Les couleurs indiquent la catégorie de classement dans lesquelles ils ont été versés.

\subsection{Concepts essentiels}

- Tous les éléments d'un itinéraire sont classés dans la même catégorie d'importance. Du point de vue du patrimoine à conserver, il est en effet plus admissible de surévaluer un objet que le contraire. Ventiler dans différentes catégories les sites d'un même itinéraire n'aurait pas seulement entraîné

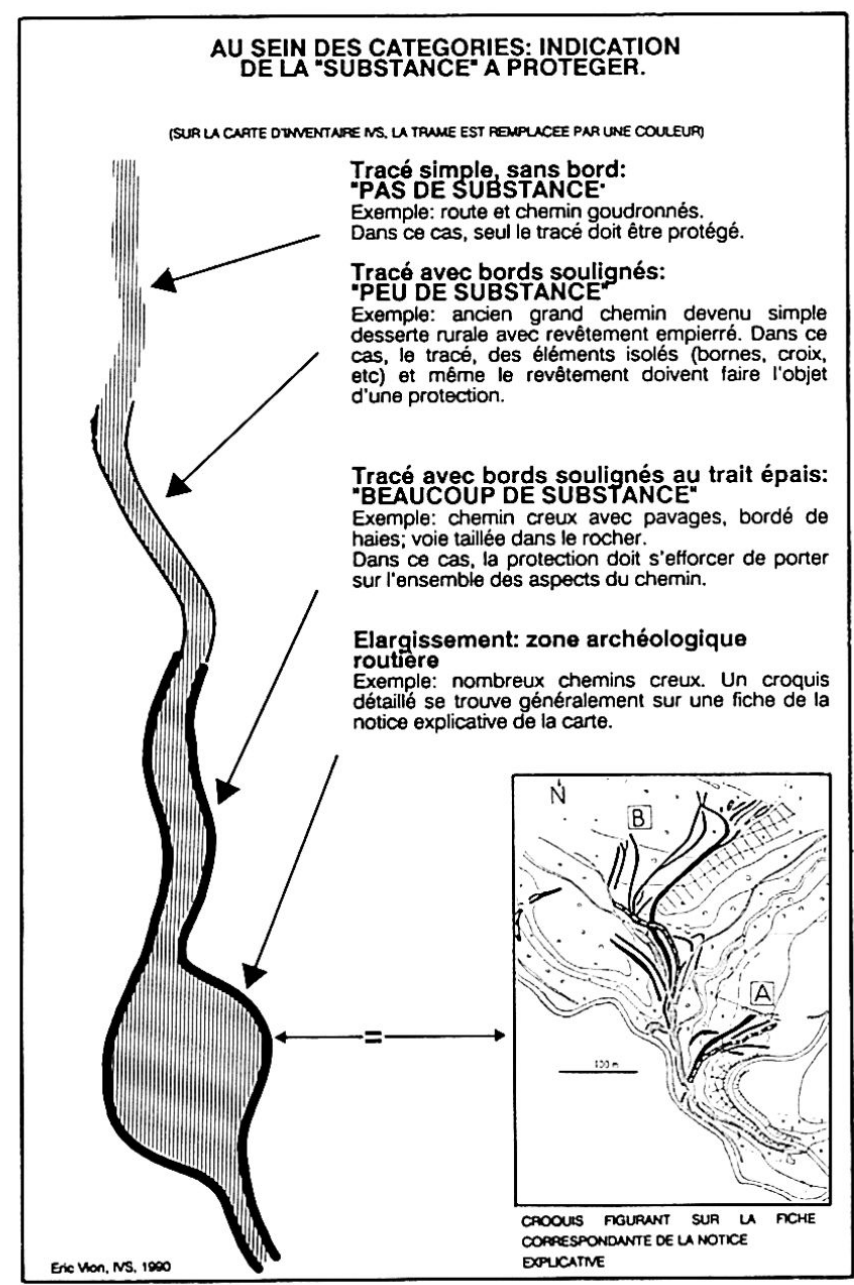

Figure 2 II a été possible d'exprimer graphiquement la relative importance des sites à protéger. Dans la même catégorie (nationale, régionale ou locale), les chemins ont, en effet, été plus ou moins modernisés et il est bon de signaler au planificateur où son attention devra être particulièrement vigilante. Du point de vue de la numérotation, les différentes "Substances" déterminent autant de segments documentés dans des fiches comportant description, commentaire, croquis et/ou photographie. 
ITINERAIRE PAYERNE - MORGES par Donneloye et Echallens disparu après 1699

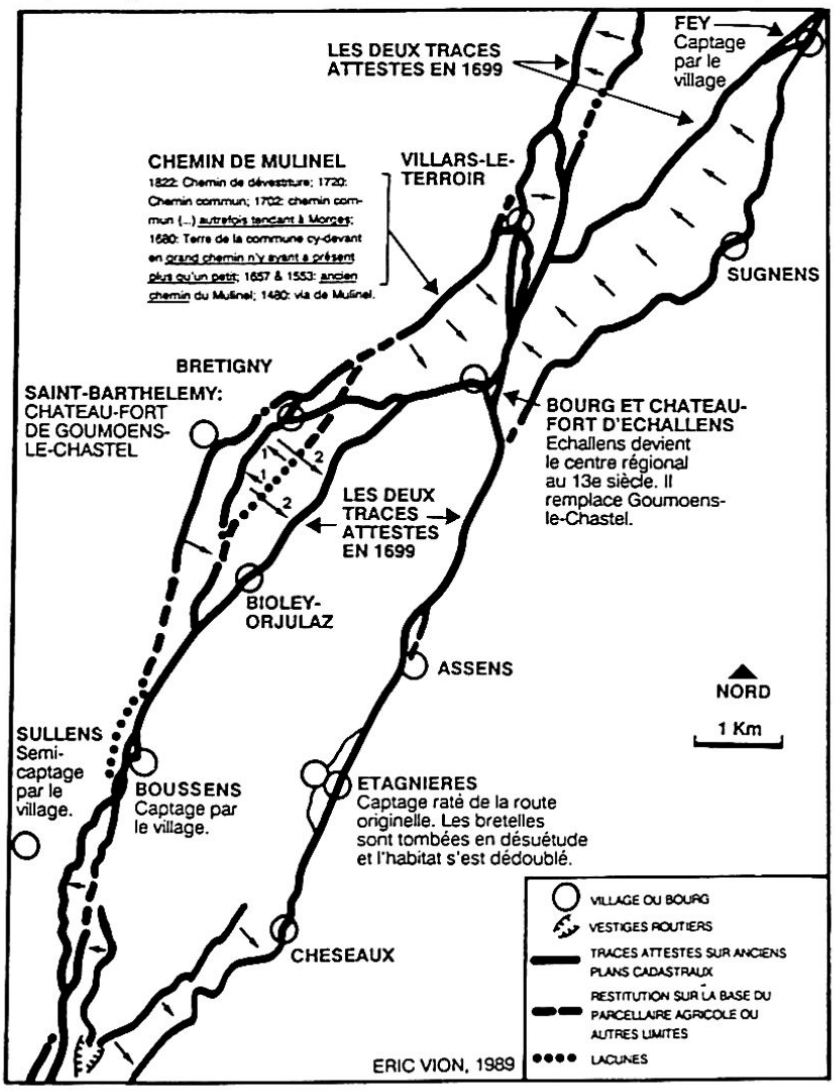

Figure 3 Voici un exemple d'itinéraire historique. C'est une grande voie de transit mais pas du tout un exemple isolé. Son histoire peut - avec prudence - être tracée jusqu'à l'époque romaine. C'est par là que les Bernois firent la conquête du pays de Vaud en 1536. II a joué un rôle important dans le transit des marchandises jusqu'à la fin du 17e siècle, date à laquelle Leurs Excellences de Berne fixèrent la circulation dans la vallée de La Broye. Sur cette carte, il faut remarquer les nombreux captages affectant le tracé originel notamment le mouvement impliqué par le développement d'Echallens qui ravit sa position à Goumoens-le Chastel à partir du $12 \mathrm{e}$ siècle. On remarquera également quelquesunes des attestations qui marquent la désuétude d'un des anciens tracés (chemin de Mulinel).

de difficiles discussions. Elle aurait surtout démembré les itinéraires dont on n'aurait plus pu suivre la cohérence. Or, du point de vue du paysage, la structure d'ensemble a souvent plus de valeur que le détail. La somme est plus que l'addition des parties.

Prenons un exemple, celui d'un itinéraire de Payerne à Morges par Echallens. Ce chemin a perdu en 1699 son importance pour le transit à travers le Pays de Vaud (VION, 1987/1). S'il est classé d'importance nationale, les aménagements du $19 \mathrm{e}$ siècle qui ont affecté les tracés subsistants le seront également, au même titre, par exemple, que les bornes routières de 1913 qui le jalonneraient.
- Seuls les chemins historiques attestés qui subsistent sont inscrits sur la carte. Les chemins protégés peuvent donc être discontinus.

- Tous les détails morphologiques des tracés ont été schématisés à l'extrême, suivant qu'ils étaient jugés pas, peu ou très consistants.

- L'itinéraire n'est connu que par une triple numérotation renvoyant aux notices. Les informations y sont organisées selon un découpage de plus en plus fin du réseau: fiches «itinéraire», "tracé», «segment».

\subsection{Les avantages et les limites}

Premier avantage, le caractère indicatif de la carte: L'importance des sites ne dépend pas du choix des planificateurs mais d'une décision de la commission nationale chargée d'évaluer les résultats de l'IVS. Le maniement de la carte ne réclame donc pas de connaissances historiques. Il n'y a pas d'interprétation à faire avant de pouvoir l'utiliser. Les catégories de classement sont celles prévues par la loi.

Le deuxième avantage, qui a été évoqué plus haut, mais qu'il faut encore souligner, est celui apporté par le concept central: le réseau des routes et chemins est présenté comme un des éléments fondamentaux de la structure du paysage. Il forme une trame vécue quotidiennement et non une collection de sites disparates et désuets.

Les défauts de la carte d'inventaire découlent directement de ses qualités. Si sa structure a été pensée pour contenter le planificateur, elle peut décontenancer l'historien ou l'amateur curieux. Dès que le réseau historique est dense - et c'est généralement le cas en plaine - sa lecture devient impossible ou très difficile. C'est bien le cas de la feuille 1223 Echallens qui a servi à élaborer le modèle de synthèse retenu par l'IVS. Tant que l'on a affaire à des experts pressés ou des aménagistes soucieux de suivre la carte, peu importe. Mais si des négociations doivent être entreprises, on a alors tout intérêt à sortir une carte des itinéraires historiques. Et les arguments ou les faits historiques devront être cherchés dans les notices. Le poids de l'information scientifique est en effet souvent essentiel.

\subsection{Le classement}

Le classement dans les trois catégories prévues par la loi - nationale, régionale, locale - ne saurait reposer uniquement sur des critères rigoureux. L'existence et le rôle même de la commission nationale l'indiquent d'ailleurs bien. Il y a des faits historiques: l'itinéraire est-il de transit? Mais, tout de suite après, il faut bien évaluer les cas particuliers. Or ils le sont tous, par les régions traversées, les époques et les villes desservies! Comment mettre sur le même plan la route cantonale de Lausanne à Yver- 
don dont la belle époque s'étend de 1820 à 1980 et l'itinéraire Morges-Yverdon dont l'apogée est bien antérieure? L'histoire fabrique des situations irréductibles. le transit, lui-même, n'a pas la même signification en 1200 ou en 1850 .

Ensuite, la notion de patrimoine est en pleine évolution depuis un siècle. Et nul doute que l'IVS, justement, en dégage un nouveau pan. Dès lors, comment s'enfermer dans des catégories rigides?

S'il a donc été admis que tous les segments d'un même itinéraire seraient inscrits dans la même catégorie, il n'en demeure pas moins que certains sites isolés peuvent faire l'objet d'un reclassement dans une catégorie supérieure. Leur morphologie ou leur rareté peut le justifier. Mais ce dernier critère est à nouveau subjectif: le contexte peut être plus ou moins large, suisse ou régional. Enfin, il est certain qu'un itinéraire aux nombreux tracés en comportera au moins quelques-uns qui seront modernisés. Ce n'est pas parce qu'une voie romaine est goudronnée que son tracé perd toute valeur.

Dans la catégorie nationale entrent donc les itinéraires de transit, des revêtements et monuments uniques ou rares (ex.: voies taillées dans le rocher avec marches et ornières; bornes armoirées avec indications de distances, ponts, etc...) et des sites exceptionnels (ex.: chemins creux profonds en très grand nombre). L'ancienneté et la morphologie d'un tracé peuvent enfin être suffisants pour l'inscription en catégorie nationale (ex.: voie rectiligne de crête évitant les villages, présumée romaine).

La catégorie régionale rassemble, en règle générale, les grands itinéraires cantonaux. La locale contient les routes intercommunales et les réseaux de desserte des champs (réseaux stellaires dans les régions à habitat groupé). Dans les régions de carrefour - par exemple, le pays de Vaud -, il faut noter, contrairement à ce que l'on pourrait attendre, la forte densité du réseau des grands chemins. Avec pour conséquence que relativement peu de chemins, entre leur origine et nos jours, n'ont connu qu'une importance strictement locale.

\section{Les recherches permettant la production de la carte d'inventaire}

Le lecteur attentif aura bien compris que la réalisation d'une carte d'inventaire IVS suppose des phases d'élaboration antérieure. Définir un but est bien sûr indispensable. Mais il faut aussi mettre au point une stratégie de travail.

\subsection{Méthodologie fondamentale}

Les recherches traditionnelles collectionnaient les parties pour en tenter l'assemblage. Aujourd'hui, la démarche est inverse: il faut partir du tout et y identifier des éléments. Les segments ne sont ainsi plus étudiés isolément mais relativement à d'autres, ce qui permet la maîtrise de l'interprétation. Si les faits historiques disparus ne peuvent être restitués par miracle, la datation est au moins relative et s'inscrit, elle aussi, dans un schéma cohérent.

Mais comment partir du réseau? Au départ, on ne connaît ni le réseau médiéval et encore moins celui de l'époque romaine ou des époques antérieures... Si l'on veut une approche globale, on ne peut donc aborder la question que régressivement. Seul le réseau actuel - ou celui des siècles immédiatement antérieurs au nôtre - peut nous fournir une base d'étude. Le réseau actuel ou récent contiendrait-il en luimême les germes de sa propre histoire? Cette hypothèse centrale est aujourd'hui vérifiée de plusieurs manières:

1. Un itinéraire considéré dans sa durée est toujours un faisceau de tracés. Il se déplace dans le temps et dans l'espace. or les tracés abandonnés ne disparaissent que très progressivement. Déclassés, ils trouvent d'autres fonctions, plus locales. Al'extrême, ils survivent sous forme de vestiges, de limites parcellaires ou de droits de passage (vion, 1990/2, fig. 1).

2. S'ils ont perdu leur fonction, les tracés gardent néanmoins leur forme caractéristique, plus stable parce qu'inscrite dans le paysage. Ces formes sont détectables et lisibles.

3. La preuve que ces formes caractéristiques détectées sont porteuses d'information est donnée par leur corrélation avec les mentions d'itinéraires anciens ou disparus tirées des archives (VION. 1989/1).

4. Vient un moment où les formes détectées ne peuvent plus être mises en relation avec des mentions d'itinéraires car elles préexistent aux archives conservées. Dès 1450 - grosso modo - on entre progressivement dans les domaines d'une archéologie routière. L'analyse du rapport entre la voie et l'habitat prend alors le relais de la récolte des mentions d'itinéraires dans les archives (VION, 1990/1).

5. La densité du réseau retrouvé s'explique au bout du compte par cet empilement progressif des chemins de toutes époques.

6. L'analyse chronologique des itinéraires donne les clefs pour comprendre cette densification.

Il y a donc des faits de structure (forme des itinéraires, densité du réseau) et une cohérence (corrélation entre formes et mentions d'itinéraires anciens, rapport entre la voie et l'habitat, chronologie interne des itinéraires) qui montrent tous que le réseau actuel est bien constitué d'un empilement de strates routières successives.

Dès lors, le problème fondamental est de savoir comment lire le réseau. Il faut bien sûr collecter des faits 


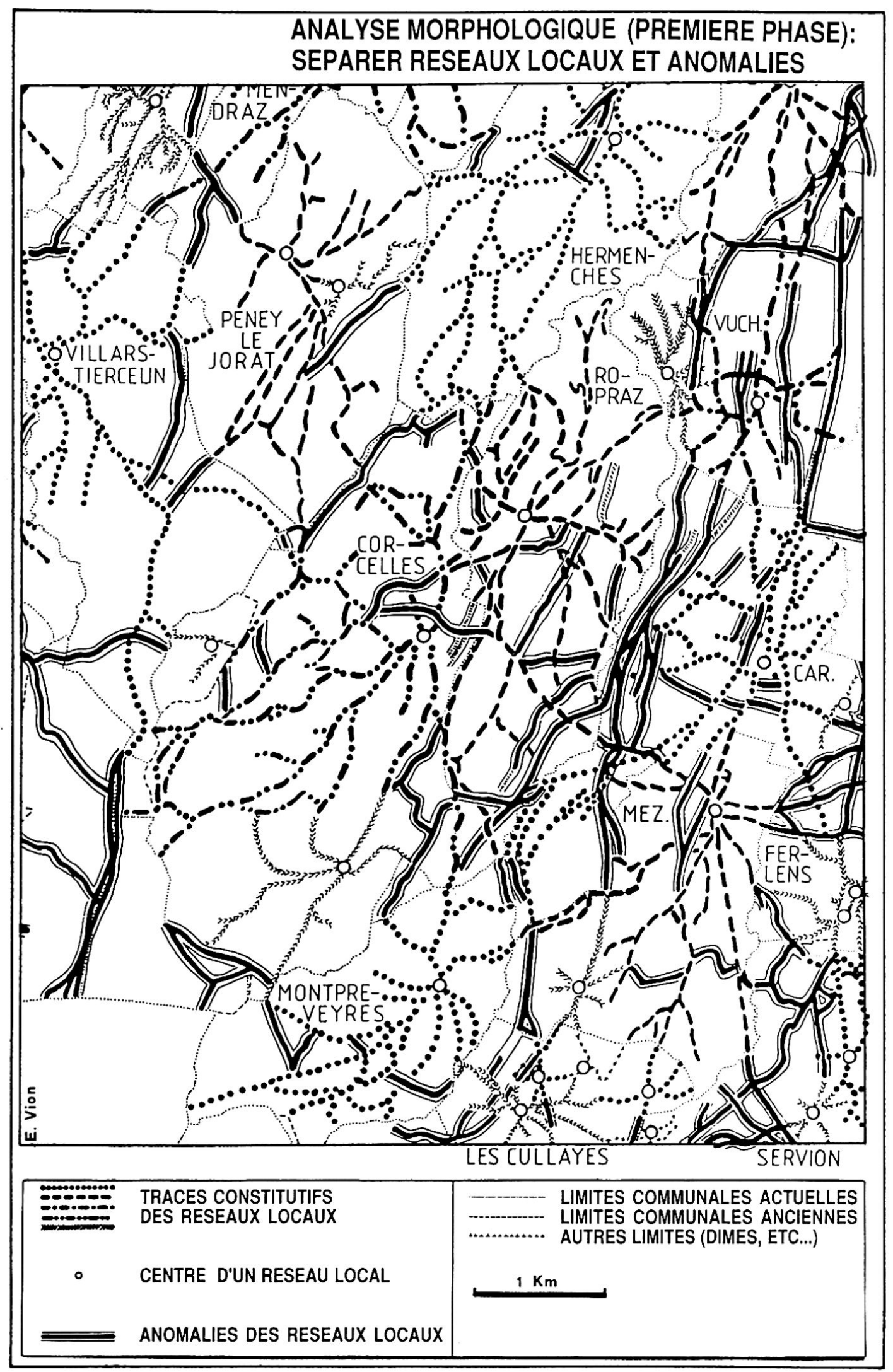

Figure 4b Les anomalies (ici, au trait épais) sont reportées sur une seconde carte et reliées par des tracés appartenant aux réseaux locaux. Morphologiquement, on a ainsi fait apparaître un réseau régional. Les recherches d'archives permettent d'établir une forte corrélation entre ce réseau et celui issu du relevé des mentions de grands itinéraires. Preuve est ainsi faite de l'intérêt d'une lecture morphologique. Quant à la densité de ce réseau, elle s'explique par le fait qu'un réseau routier est constitué de strates successives (les mêmes archives montrent que seuls environ $7 \%$ des grands chemins sont en pleine activité). C'est la preuve qu'une analyse morphologique et archéologique du réseau est possible. 


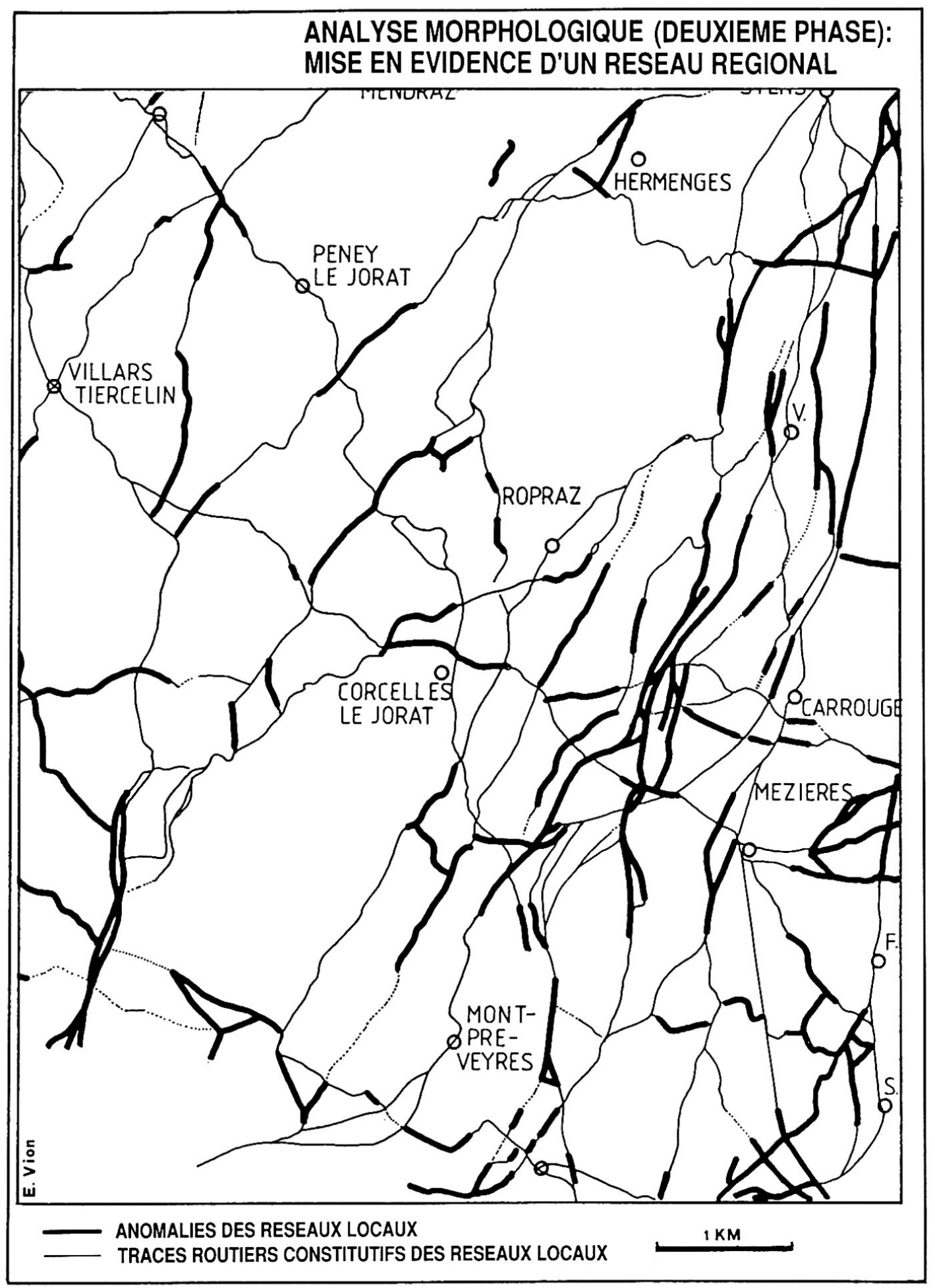

Figure 5 Cette carte compile l'ensemble des données récoltées: tracés issus de la lecture morphologique, attestations d'archives, levés de terrain. La densité est extrême, de l'ordre de 5-6 km de chemins anciens au km². Elle témoigne bien de la longue accumulation dans le paysage des tracés successifs des itinéraires. Elle illustre bien le pouvoir structurant des chemins sur l'espace. La tâche de l'historien sera de débrouiller cet écheveau en y individualisant des itinéraires, c'est-à-dire des faisceaux de tracés (cf. fig. 3). Ensuite viendra une ultime étape, celle de la reconstitution de l'histoire interne de l'itinéraire. 
tant sur le terrain que dans les archives. Mais il faut surtout faire une lecture d'ensemble afin de débrouiller la densité accumulée par des millénaires de circulation.

\subsection{Appréhender et dater la forme des réseaux}

L'analyse morphologique a pour but de faire apparaître des formes aux caractéristiques différentes. C'est une opération graphique simple.

Sur le plateau vaudois, l'habitat est généralement groupé en villages autour desquels s'est développé un réseau stellaire de chemins. Aux confins des terroirs, ils font jonction avec ceux des communes adjacentes ou se perdent dans les champs et les bois qu'ils desservent. J'ai nommé ce groupe de tracés «réseau local» car il dépend toujours d'un habitat proche.

Il y en a des bien réguliers parce que dépendant d'une localité bien centrée sur son territoire. D'autres s'inscrivent dans une topographie contraignante et sont considérablement déformés. Il faut alors considérer que le plus court chemin n'est pas formément la ligne droite. Les petites localités fortifiées ont très souvent un réseau difforme: la facilité de la défense réclame un escarpement par essence marginal dans l'univers cultivé des hommes.

Plusieurs réseaux locaux, dépendant de plusieurs hameaux, peuvent se partager inégalement un même territoire communal. Si l'habitat dispersé vient à prédominer, ils tendent à s'atomiser et sont plus difficiles à définir parce que noyés dans une trame régionale qui a de fortes chances d'être antérieure.

Longs ou courts, les tracés qui ne s'intègrent pas aux réseaux locaux sont assez nombreux. Nous en avons noté en moyenne presque un par kilomètre carré (CN 1223). Ces «anomalies» des réseaux locaux répondent à une autre logique dont les mentions d'itinéraires des archives livrent la clef. Elles marquent avant tout un réseau supra-local - c'est-à-dire - régional et, dans une beaucoup plus faible mesure, l'accès à des installations industrielles - moulins, tuillières ou à des bâtiments isolés - églises, châteaux -, des extensions territoriales d'une commune sur une autre et des organisations cadastrales anciennes (VION, 1989/1).

La disjonction entre la voie et l'habitat rural de l'Antiquité est un fait capital qui a été souligné (CHEVALLIER, 1972, p. 131; CHOUQUER, 1982, p. 341-2). Mais nos villages - jusqu'à preuve du contraire sont des établissements du Haut Moyen Age. leur origine peut rester controversée... Le regroupement de l'habitat rural dispersé du $2 \mathrm{e}$ siècle est néanmoins incontestable, ne serait-ce que parce que les villae sont plus nombreuses que les villages (KRAUER, 1984, vol. 1, p. 61). Il n'est même pas nécessaire de supposer que la plupart des villages sont édifiés sur d'anciennes villae. Si continuité il y a eu, c'est d'abord dans le mode de fondation. La moitié environ des villages du plateau vaudois est à l'origine bâtie à l'écart d'un axe de communication régionale.

Il faut en tirer la conséquence. Si la mise en place des réseaux locaux a fatalement accompagné celle des villages, la relation chronologique entre ces derniers et le réseau régional doit être pesée.

Simultanéité et postériorité doivent être écartées. La redistribution de l'habitat antique introduit en effet justement une rupture par rapport à l'ordre antérieur. Un long mouvement de capture des grands chemins par les villages s'amorce. Perceptible dans les archives depuis le Moyen Age, il s'accélère après 1700 avec les modernisations routières et la pression démographique sur la terre. Seule la création de bretelles d'évitement, après 1950 , renouera avec la tradition.

Le réseau des grands chemins est donc antérieur. Pourtant, à l'échelle régionale, les voies d'Empire n'ont pas dû être si nombreuses... Dès lors l'origine du fonds du réseau routier se perd dans la nuit des temps. La structure a traversé l'époque romaine comme les suivantes (VION. 1989/1).

\subsection{Stratégie de recherche}

Première étape: Choisir un réseau sur lequel travailler.

Il faut pouvoir disposer d'un réseau «complet». La plus récente des cartes peut faire l'affaire. Mais elle comportera de nombreux "parasites», résultat des travaux d'aménagements modernes. On aura donc tout intérêt à utiliser les cartes détaillées les plus anciennes possibles, pour autant qu'elles restituent l'entier du réseau routier. Les premières éditions de l'atlas Siegfried, les minutes de la carte Dufour sont une bonne base. Dans certains cas, comme celui du pays de Vaud, le relevé des plans cadastraux permet de remonter plus haut: au 18e siècle, voire à la seconde moitié du $17 \mathrm{e}$ siècle, c'est-à-dire à une période antérieure aux grandes modernisations.

Deuxième étape: Ce réseau fera l'objet d'un traitement graphique qui séparera le réseau régional des réseaux locaux.

Troisième étape: Le dépouillement des sources foncières (plans cadastraux d'abord puis reconnaissances féodales) permettra la récolte des attestations d'itinéraires jusqu'au Moyen Age.

Quatrième étape: Réseau morphologique et réseau bâti sur la base des mentions d'itinéraires seront fondus.

Cinquième étape: Le réseau obtenu sera très dense (CN 1223: 5 à $6 \mathrm{~km}$ de chemins au $\mathrm{km}^{2}$ ). Tous les tracés du réseau régional devront être distribués dans 


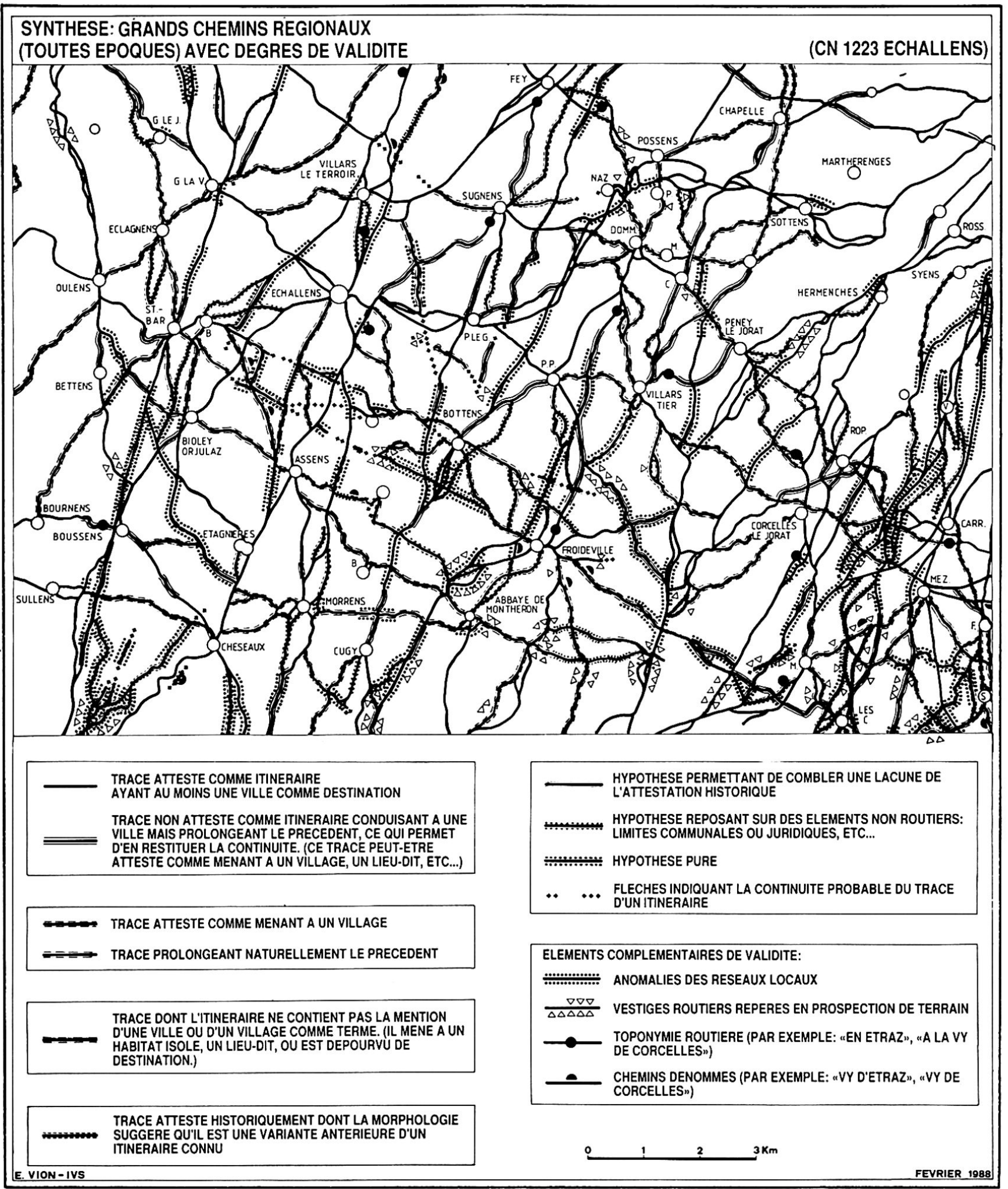

Figure 4a Dans les régions à habitat groupé, il est aisé de mettre en évidence autour des villages un réseau local, le plus souvent en étoile. Une fois individualisés, les réseaux locaux laissent des anomalies que nous avons soulignées. Elles marquent, morphologiquement, le passage de chemins d'un réseau supra-local, c'est-à-dire régional. S'il faut noter que ce marquage est intermittent, il faut aussi relever sa densité. Nous sommes en effet ici entre Lausanne et Moudon (Partie est de la CN 1223 - Echallens), c'est-à-dire sur un des axes principaux trans-helvétique entre Genève et le Rhin. 
des faisceaux qui sont la réalité morphologique des itinéraires.

Sixième étape: Il faudra reconstituer l'histoire interne de tous les itinéraires.

On disposera ainsi d'une carte des itinéraires historiques. La comparaison entre celle-ci et le réseau actuel sera à la base de la construction de la carte d'inventaire IVS. Les relevés de terrain permettront enfin la segmentation morphologique des tracés retenus.

\section{Zusammenfassung}

Seit 1984 entsteht auf der Grundlage des Natur- und Heimatschutzgesetzes ein vom Bund in Auftrag gegebenes «Inventar historischer Verkehrswege der Schweiz» (IVS). Das Aufnehmen von isolierten Einzelobjekten (Hohlwege, Distanzsteine usw.) hat sich dabei schnell als ungenügend erwiesen, wird es doch den Bedürfnissen des heutigen Landschaftsschutzes nicht gerecht. Vielmehr gilt es, das historische Verkehrsnetz zu untersuchen und ganze, durchgehende Verbindungen in die künftigen Planungen einzubeziehen.

Aber diese Art von Heimatschutz ist aus einer neuen Geländewissenschaft hervorgegangen. Der Begriff des Heimatschutzes und jener der wissenschaftlichen Forschung sind darin untrennbar verbunden.

Der Autor beschreibt Vorarbeiten aus der Westschweiz, die zusammen mit Erfahrungen aus anderen Gebieten zur Erstellung der Inventarkarte des IVS geführt haben.

Die Inventarkarte ist vor allem auf die Bedürfnisse ihrer Benützer, insbesondere der Raumplaner, ausgerichtet. Sie liefert nicht eine Sammlung von Objekten einer überprägten Vergangenheit, sondern zeigt die historischen Wurzeln des aktuellen, beste- henden Wegnetzes. Die schutzwürdigen Strecken sind gemäß Natur- und Heimatschutzgesetz in die drei Kategorien von nationaler, regionaler und lokaler Bedeutung eingeteilt.

\section{Bibliographie}

BRAUDEL, F. (1985): La Méditerranée et le monde méditerranéen à l'époque de Philippe II, Armand Colin, Paris, 4 éd.

CHEVALLIER, R. (1972): Les voies romaines, Armand Colin, Paris.

CHOUQUER, G. (1982): Les cadastres romains, approche morphologique et problèmes, Thèse, Besançon, pp. 341-2.

KRAUER, A.-P. (1984): Recherches sur l'occupation du territoire à l'Epoque romaine et au Haut Moyen Age, Mémoire, Université de Lausanne.

LEPETIT, B. (1984): Chemins de terre et voies d'eau. Réseaux de transports et organisation de l'espace en France 1740-1840, Ed. de l'Ecole des Hautes Etudes et Sciences sociales, Paris.

VION, E. (1987/1): Le contexte routier du canal, la place de la liaison Yverdon - Morges dans l'histoire des communications vaudoises. Dans: Canal d'Entreroches, Cahiers d'archéologie romande no 33, pp. 83-98.

VION, E., et alii (1987/2): Chemins du pays de Vaud, Recueil des articles de la Gazette de Lausanne.

VION, E. (1989/1): L'analyse archéologique des réseaux routiers: une rupture méthodologique, des réponses nouvelles. Dans: Paysages découverts no 1, Lausanne, pp. 67-99.

VION, E. (1989/2): L'archéologie du territoire: étudier le passé pour gérer le présent. Dans: Paysages découverts no 1, Lausanne, pp. 9-21.

VION, E. (1990/1): Itinéraires et lieux habités: les deux pôles de l'analyse archéologique des réseaux routiers. Dans: Actes du colloque de Belley, 3-4 juin 1989, à paraitre dans Caesarodunum, Université de Tours, 1990.

VION, E. (1990/2): Anciens chemins et mémoire du paysage, cours de gestion du paysage 1989, Ecal, Lausanne, à paraître. 